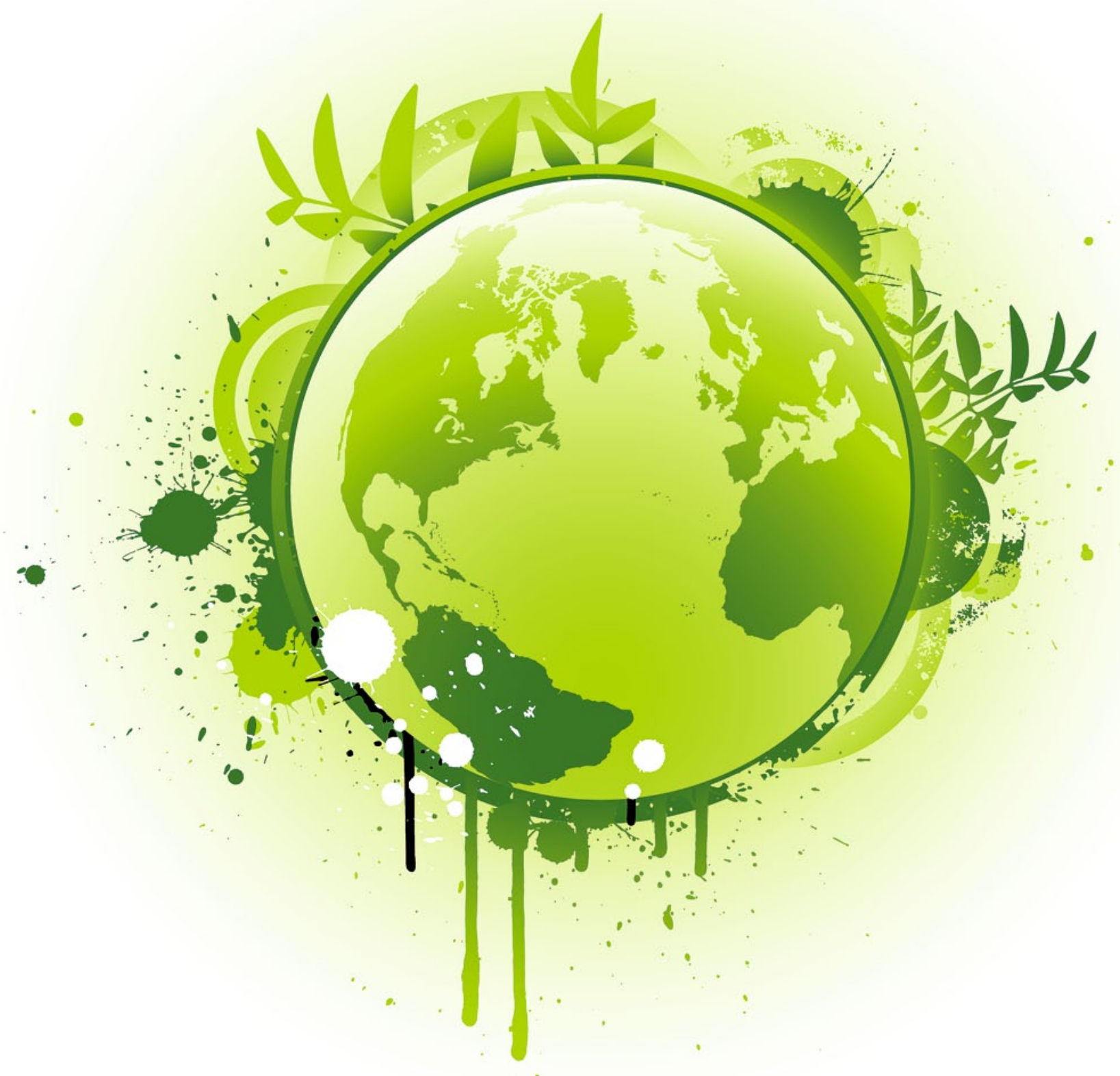

\title{
Beyond Oil? Transformation in eine post-fossile Gesellschaft
}

Seit der im Jahr 1972 erschienenen Studie „Grenzen des Wachstums“ hat sich die ökologische Debatte stark auf die Gefahr der Verknappung von Rohstoffen konzentriert. Die neuere Umweltforschung zeigt, dass noch weit vor dem Ende der fossilen Rohstoffe andere Umweltgrenzen überschritten werden. Der Beitrag widmet sich den sich daraus ergebenden Konsequenzen für Unternehmen.

Von Prof. Dr.-Ing. Dr. Christian Berg und Prof. Dr. Uwe Schneidewind 
$\mathrm{D}$ Erdölnachfrage hat in den vergangenen Jahrzehnten massiv zugenommen, vor allem im Zuge des Aufschwungs der Schwellenländer. Lange Zeit konnten die Fördermengen der steigenden Nachfrage entsprechend ebenfalls wachsen - einer steigenden Nachfrage stand ein wachsendes Angebot gegenüber. „Peak Oil“ bezeichnet den Punkt, an dem keine weitere Angebotsausweitung mehr erfolgt - bei weiterhin hoher Nachfrage und damit erheblich steigenden Preisen. Allerdings hat sich die Frage, wann dieser Peak der globalen Produktion erreicht sein wird, durch vermehrte Förderung unkonventioneller Vorkommen wie Schiefergasen oder Teersanden relativiert, denn durch diese wird die global verfügbare Menge noch auf längere Zeit gesteigert werden können. Insbesondere die in Nordamerika vorkommenden unkonventionellen Vorräte verändern die Projektionen der Fördermengen erheblich. So geht die Internationale Energieagentur inzwischen (2012) davon aus, dass die USA bis 2015 zum größten Gas- und bis 2017 zum größten Ölproduzenten der Welt aufsteigen und sich in diesem Zuge zu einem NettoExporteur von Öl und Gas wandeln wird [12]. In einem Report von 2012 sprach die Citigroup sogar schon vom „Tod der Peak-Oil-Hypothese“ [5].

\section{»Das Fracking erfordert große Mengen an Energie, Wasser und Chemikalien und führt zu erheblichen Gefahren für das Trinkwasser. «}

Gegenwärtige Schätzungen gehen davon aus, dass eine weitere (moderate) Steigerung der Erdölproduktion bis 2036 möglich ist [2, S. 20] und dass fossile Energieträger auch 2030 noch etwa $80 \%$ des globalen Primärenergiebedarfs decken werden [8, S. 7]. Die Frage ist allerdings, zu welchem Preis. Denn das „hydraulic fracturing“ („Fracking“), das zur Erschließung der unkonventionellen Reserven angewendet wird, ist mit erheblichem Aufwand verbunden - finanziell wie auch ökologisch. Das Fracking erfordert große Mengen an Energie, Wasser und Chemikalien und führt zu erheblichen Gefahren für das Trinkwasser; wegen des hohen Energieeinsatzes hat es außerdem eine noch schlechtere $\mathrm{CO}_{2}$ Bilanz (ca. 10 bis $15 \%$ ) [11, S. 8].

Die Ära billigen Erdöls wird deshalb wohl bald zu Ende zu gehen, wie eine Shell-Studie es beurteilt: 2015 werde die wachsende Förderung von leicht zugänglichem Öl und Gas nicht mehr ausreichen, um die absehbare Zunahme im Bedarf zu decken [19, S. 8]. Dennoch werden die absehbaren Preisanstiege allein nicht ausreichen, um den Ausstieg aus fossilen Energieträgern so zu beschleunigen, wie es für die Lösung anderer Umweltherausforderungen nötig wäre.

\section{Klimawandel}

Denn dass Erdöl knapper, teurer und dreckiger wird, ist nicht der einzige Grund, um die Ölabhängigkeit zu überdenken. Viel wichtiger erscheint die Tatsache, dass die bei der Verbrennung freigesetzten Treibhausgase den Klimawandel beschleunigen. Wir befinden uns angesichts dessen in einer geradezu schizophrenen Situation: Auf der einen Seite hat die Weltgemeinschaft die Bedrohung durch den Klimawandel erkannt und sich auf der Konferenz von Cancún 2010 auf das Ziel verständigt, die globale Erwärmung gegenüber dem vorindustriellen Niveau langfristig auf weniger als zwei Grad Celsius zu begrenzen. Auf der anderen Seite sind wir von einer Realisierung dieses Ziels weiter entfernt denn je. Die $\mathrm{CO}_{2}$-Emissionen nehmen weiterhin $\mathrm{zu}, 2011$ erreichten die globalen Emissionen ein neues Allzeithoch [13]. Wenn nicht sehr bald substanzielle Fortschritte erzielt werden, ist langfristig eine Erwärmung von 5 Grad Celsius oder mehr zu erwarten, was wiederum schon zum Ende dieses Jahrhunderts einen Anstieg des Meeresspiegels um bis zu einem Meter, in den folgenden Jahrhunderten noch um erheblich mehr bedeuten würde [23, S. 5].

Der Übergang in ein postfossiles Zeitalter muss und wird daher global noch stärker politisch flankiert werden als bisher. Das hat weitreichende Konsequenzen für die politische Rahmensetzung, aber auch für die strategische Steuerung von Unternehmen.

\section{Treiber für den Übergang ins postfossile Zeitalter}

Ein zentrales Problem bei der Bekämpfung des Klimawandels ist, dass Emissionen keinen allgemein anerkannten Preis haben und damit auch nicht den Marktmechanismen unterworfen sind. Gegenwärtig ist zu erleben, dass regionale Initiativen in vielen Weltregionen Emissionen mit einem Preis belegen - obwohl es noch nicht gelungen ist, ein international verbindliches Abkommen über die Reduktion der Treibhausgase nach Maßgabe des Kyoto-Protokolls zu verabschieden. Seit 2005 gibt es das Emissionshandelssystem in der EU, seit 2008 in Neuseeland, seit 2012 erhebt Australien eine $\mathrm{CO}_{2}$-Steuer, und einige US-Staaten haben ein verbindliches Emissionsreduktionsprogramm für den Energiesektor eingeführt. Süd-Korea plant die Einführung eines Emissionshandelssystems für 2015, China hat nicht nur ambitionierte Ziele für die Senkung der $\mathrm{CO}_{2}$-Intensität, es wird in zwei Provinzen und fünf Städten auch ein Emissionshandelssystem pilotieren. Das gemeinsame Ziel all dieser Maßnahmen ist, Emissionen in Marktmechanis- 
men einzubinden: $\mathrm{CO}_{2}$ bekommt einen politisch festgesetzten, steigenden Preis.

Die politische Dynamik, mit der sich weitere große Staaten einem verbindlichen Klimaregiment unterwerfen, ist noch schwerer abzuschätzen als die mengenmäßige Verknappung von Rohstoffen. Veränderungen werden hier z. T. über einzelne Schlüsselereignisse katalysiert. Die Reaktorkatastrophe von Fukushima und die Auswirkungen auf die deutsche Energiepolitik sind ein eindrucksvolles Beispiel dafür. Die Auswirkungen des Wirbelsturms Sandy und vergleichbarer Ereignisse in der Zukunft auf die amerikanische Klimapolitik lassen sich kaum prognostizieren. All dies stellt neue Anforderungen an die Risikovorsorge von Unternehmen, die in ihren Produktions- und Produktstrukturen noch stark auf fossile Energien setzen.

Carbon Bubble? Verbrennung fossiler Ressourcen unvereinbar mit Klimazielen

Besonders plastisch wird die Bewertungsherausforderung im Hinblick auf die Bewertung von Energie-Unternehmen mit umfassenden Verfügungsrechten an fossilen Rohstoffen:

Um das 2-Grad-Ziel zu erreichen, dürfen im Zeitraum von 2000 bis 2050 insgesamt nicht mehr als etwa $886 \mathrm{Gt} \mathrm{CO}_{2}$ ausgestoßen werden - das folgt aus dem sog. „Budgetansatz“ [4; 20]. Je später die Reduktion beginnt, desto rascher müsste der Null-Emissions-Zustand erreicht werden. Wäre es möglich, sofort mit der Reduktion zu beginnen, würde eine
Reduktion von $4 \%$ p. a. bis 2040 genügen; beginnen wir erst 2020, müsste die Absenkung schon $9 \%$ betragen. Wenn wir weitermachen wie bisher, ist das Budget bereits 2032 ausgeschöpft [20].

Blickt man angesichts dieser Zahlen auf die heutigen Reserven fossiler Energieträger, stellt sich die Frage, ob diese jemals genutzt werden können. Allein die mit den heute bekannten Ölreserven verbundenen Emissionen würden ausreichen, um das globale Emissionsbudget auszuschöpfen. Nimmt man noch die Kohle- und Gas-Reserven hinzu, kommt man mit insgesamt $2.800 \mathrm{Gt}$ auf ein Vielfaches der Menge, die das Emissionsbudget erlaubt [4, S. 6].

\section{Unternehmensbewertungen auf dem Prüfstand}

Die britische Carbon-Tracker-Initiative schlussfolgert deshalb, dass das Emissionsbudget faktisch zu einem Rückgang in der Nachfrage führen wird und eine Neubewertung der Vermögenswerte von Unternehmen im Bereich fossiler Energieträger erforderlich machen würde. Nach der ImmobilienBlase würden die Finanzmärkte nun eine Kohlenstoff-Blase vor sich herschieben, da die Emissions-Äquivalente der fossilen Energiereserven in die Unternehmensbewertung bisher noch kaum eingingen [4].

Eine solche Schlussfolgerung wird durch zwei Entwicklungen untermauert: Zum einen zeichnet sich schon heute $a b$, dass - infolge der Finanz- und Schuldenkrise - die Werthaltigkeit längerfristiger Investitionen künftig noch kritischer

\section{Abb. 1 | Emissionspotenziale fossiler Quellen und globale $\mathrm{CO}_{2}$-Budgets}

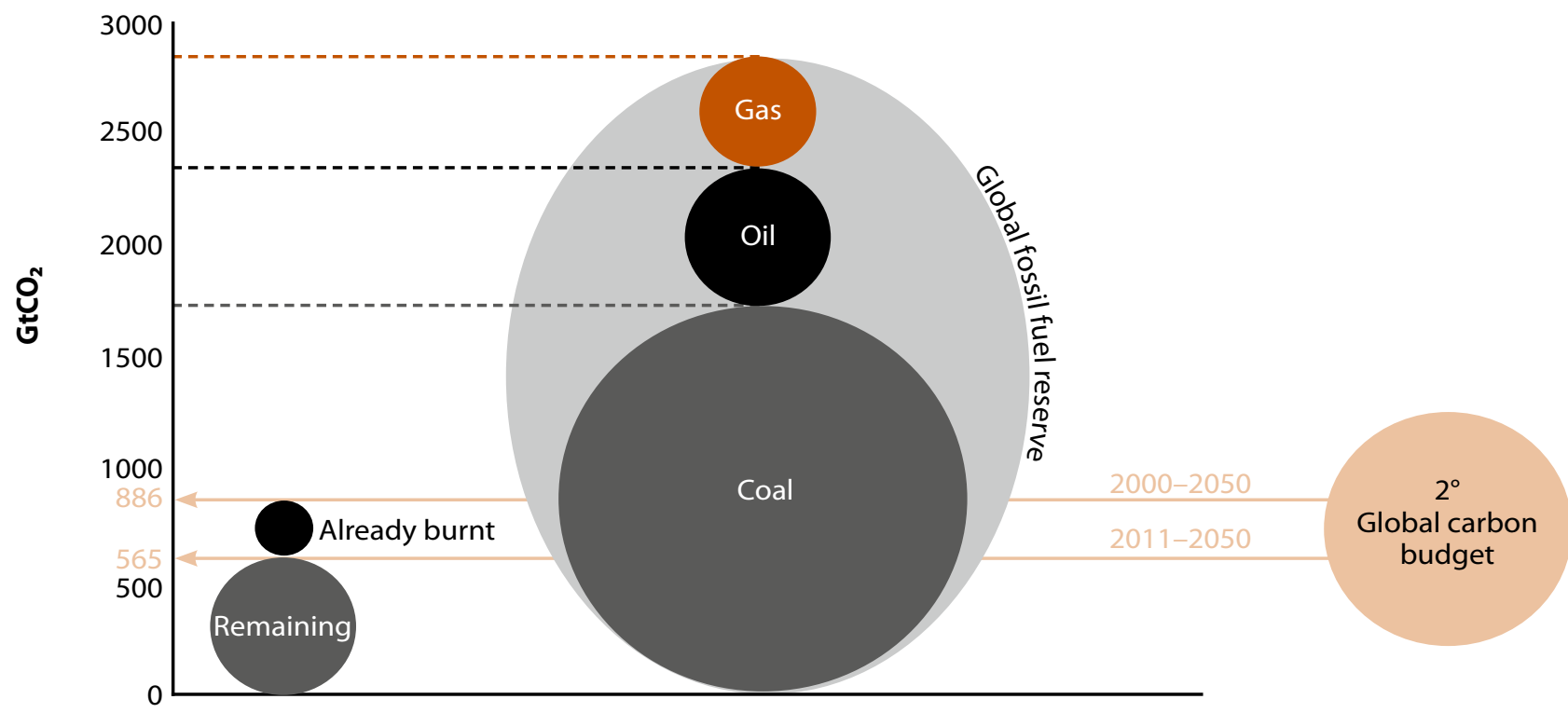


überprüft werden wird. Zum anderen erweisen sich die Finanzberichte börsennotierter Unternehmen zunehmend als unzureichend bzgl. des Ausblicks und der künftigen Markterwartungen. Die Deutsche Schutzvereinigung für Wertpapierbesitz urteilte jüngst, die Prognosen der Geschäftsberichte seien „oft wenig aussagekräftig und damit für Anleger schlicht nutzlos“ [6].

Weltweites Bewusstsein für Dringlichkeit des Klimawandels

Letztlich werden politische Entscheidungen durch Veränderungen von Werthaltungen in Gesellschaften getrieben. Hier ist mit Blick auf die Wahrnehmung des Klimawandels global einiges in Bewegung:

Der Klimawandel wird weltweit von einer großen Mehrheit der Bevölkerung als ernstes oder sehr ernstes Problem erkannt. Das geht aus dem sog. World Value Survey hervor, der größten internationalen sozial-empirischen Erhebung zur Veränderung von Wertemustern. Im fünften Turnus

\section{"Allein die mit den heute bekannten Ölreserven verbundenen Emissionen würden ausreichen, um das globale Emissionsbudget auszuschöpfen. "}

(2005 bis 2008) wurden mehr als 60.000 Menschen in 49 Ländern befragt. Auf die Frage „Wie ernst ist der Klimawandel als globales Umweltproblem?“ antworteten 89,3\% mit „sehr ernst“ oder „ernst“ [21, S. 75] (andere Antwortmöglichkeiten waren: „nicht sehr ernst“, „überhaupt nicht ernst“, „weiß nicht“). Eine Mehrheit der Befragten (54,6\%) spricht sich sogar dafür aus, dass „dem Umweltschutz mehr Aufmerksamkeit geschenkt werden sollte, auch wenn dadurch das Wirtschaftswachstum sinkt und Arbeitsplätze verloren gehen“" [21, S. 76].

Zwar haben diese Mehrheiten noch nicht dazu geführt, dass von politischer Seite ein internationales Post-Kyoto-Abkommen ermöglicht wurde. Sie zeigen aber, dass der Klimawandel durchgängig als globales Problem erkannt wird. Damit ist die wohl wichtigste Voraussetzung erfüllt, dass diese Einschätzungen auch politisches Handeln ermöglichen, was angesichts der erforderlichen Dringlichkeit ggf. auch innerhalb kurzer Zeit große Veränderungen mit sich bringen könnte. Beispiele für gravierende Veränderungen innerhalb kurzer Zeit hat es in den vergangenen Jahrzehnten wiederholt gegeben. Niemand hatte etwa damit rechnen können, dass der ehemalige Ostblock oder die diktatorischen Regime Nordafrikas innerhalb weniger Jahre zerfallen würden.

\section{Globale Vernetzung - Trigger rascher Veränderungen}

Die Dynamik gesellschaftlicher und politischer Umbrüche im internationalen Maßstab wird dabei durch die globale informationstechnische Vernetzung befördert.

Die Vernetzung unserer Welt hat seit den Anfängen von Telegraphie und Telefon vor 150 Jahren mit atemberaubender Geschwindigkeit zugenommen. Es dauerte 38 Jahre, bis 50 Millionen Amerikaner Radio hatten, beim Fernsehen waren es 13 Jahre, beim Internet 5 Jahre [22, S. 42]. Heute nutzen weltweit gut ein Drittel der Menschen das Internet, etwa eine Milliarde sind in sozialen Netzen aktiv [10]. Massenmedien, Internet, Mobiltelefonie und soziale Medien - sie alle tragen dazu bei, dass sich Informationen in rasanter Geschwindigkeit ausbreiten, dass sich Meinungen bilden, Stimmungen rasch ändern und Menschen in einer bisher unbekannten Weise organisieren können. Mit Blick auf den Klimawandel lässt sich über mögliche Folgen nur mutmaßen; es scheint aber durchaus denkbar, dass mit zunehmender Sichtbarkeit der Folgen globaler Erwärmung auch der Handlungsdruck für Politik und Wirtschaft zunehmen wird. Sollte die Arktis tatsächlich noch in diesem Jahrzehnt eisfrei werden, könnten zum Beispiel Bilder von Eisbären, deren Lebensraum buchstäblich verschwindet, eine starke emotionale Botschaft um die Welt senden und damit in kurzer Zeit eine Stimmung erzeugen, die einen erheblichen Druck auf Politik und Wirtschaft ausüben wird, um die Bemühungen zur Emissionsreduzierung deutlich voranzutreiben.

Zusammengefasst: Regierungen beginnen zu handeln, die vollständige Verbrennung fossiler Reserven ist unvereinbar mit den politisch vorgegebenen Zielen, die Bevölkerung hat das Problem erkannt, und durch die neuen Medien sind die Bedingungen für rasche Veränderungen verbessert worden.

\section{Merkmale post-fossilen Wirtschaftens}

Die skizzierten Entwicklungen lassen es vernünftig erscheinen, sich früh mit den Merkmalen post-fossilen Wirtschaftens auseinanderzusetzen. Eine solche Wirtschaft wird typische Merkmale aufweisen, die sich bereits in Ansätzen erkennen lassen.

\section{Hohe Preise für Energie und Emissionen}

Die Emission von Treibhausgasen wird mit steigenden Kosten einhergehen. Da zugleich die Zeit des billigen Öls und Gases zu Ende geht, lohnt sich Energiesparen doppelt: durch geringere Kosten für Beschaffung wie auch für Emissionen. „Carbon“ wird in manchen Industrien zur zweiten Währung, überall dort, wo fossile Energien eine wichtige Rolle spielen. 
Die Anreize zur Optimierung von Transport- und Logistikprozessen werden besonders groß sein, denn Verkehr und Transport sind gegenwärtig noch ganz überwiegend auf fossile Energieträger angewiesen.

Die Strompreise werden durch den starken Ausbau der erneuerbaren Energien mit erheblichem Investitionsbedarf in Deutschland zwar in den nächsten Jahren weiter steigen, ab Mitte der 2020er- Jahre aber wegen des dann hohen Anteils der erneuerbaren Energien sinken. [9] Der Umbau des Energiesystems ist dabei mit Innovationschancen für viele Industrien verbunden [18]. Je nachdem, wie sich die Emissionspreise bis dahin entwickelt haben werden, könnte daraus ein deutlicher Wettbewerbsvorteil für die deutsche Wirtschaft resultieren, weil deutlich weniger Emissionskosten und dann sogar sinkende Stromkosten zu erwarten sind.

Um die Kosten für Unternehmen in der Übergangszeit überschaubar zu halten, zugleich aber auch Anreize zur Verbrauchsreduktion zu geben, wäre es sinnvoll, die Unternehmensbesteuerung zu verlagern, um den Produktionsfaktor Arbeit günstiger, den Verbrauch von Energie, Ressourcen und die Inanspruchnahmen von Flächen dagegen teurer werden zu lassen. Entsprechende Vorschläge hat eine Expertengruppe der Bundesregierung bereits gemacht [15, S. 84 ff.].

\section{Lokale Beschaffung}

Mit höheren Energiepreisen, (höheren) Preisen für Emissionen und gestiegenem Bewusstsein rücken logistische Optimierungen stärker in den Blick - und eröffnen zudem unter dem Stichwort „lokale Beschaffung“ auch ökologische Vorteile und entsprechende Marketingpotenziale. Überall dort, wo Transport- und Logistikprozesse einen wichtigen Bestandteil der Wertschöpfung ausmachen, können Optimierungen in diesem Bereich zum Wettbewerbsvorteil werden.

\section{Mehr Dienstleistungen, weniger Verbrauch und} Kreislaufwirtschaft

Dass in der Netzwerkgesellschaft Zugang wichtiger ist als Besitz, wusste Jeremy Rifkin schon vor Jahren: „In einer Ökonomie, deren einzige Konstante der Wandel ist, macht es wenig Sinn, Besitz anzuhäufen “ [17, S. 13]. Das bedeutet Zugang zu Information anstatt Besitz von Büchern, Zugang zu Mobilität anstatt Besitz von Autos, Zugang zu Wertschöpfung, Dienstleistungen und Annehmlichkeiten, aber keine Anhäufung von physischem Besitz. Wenn zudem die Energie- und Emissionskosten steigen, gibt es zusätzliche Anreize, um ressourcenschonende Dienstleistungsmodelle zu entwickeln. Schon heute gibt es Beispiele erfolgreicher Geschäftsmodelle, bei denen statt Produkten Dienstleistungen verkauft werden. Xerox verkauft schon seit Jahrzehnten nicht Fotokopierer, sondern Dienstleistungen. Patagonia hat das Ideal einer Kreislaufwirtschaft auch in der Bekleidungsindustrie einge- führt und fordert dazu auf, vor dem Kauf eines Kleidungsstücks sorgsam zu überlegen, ob dies wirklich erforderlich ist. „Don’t buy this Jacket!“ Patagonia will mit langer Haltbarkeit der Artikel, einem Reparaturangebot, Empfehlungen für die Weiterverwendung bzw. Aufbereitungskonzepten für alte Fasern den Materialverbrauch senken. [14] Künftig werden es auch immer mehr Online-Tauschbörsen ermöglichen, die Nutzungsdauern von Produkten erheblich zu verlängern und den Konsum zu reduzieren. Die jüngst gegründete Plattform „yerdle“, die mit Bedacht am „Black Friday 2012“ gegründet wurde (also am Tag nach Thanksgiving, einem der wichtigsten Einkaufstage in den USA), wirbt mit dem Motto: „Sharing is more fun than shopping" [24].

\section{Transparenz und Zusammenarbeit entlang von Lieferketten}

Um Produkte und Dienstleistungen in einer ganzheitlichen Weise und über den gesamten Lebenszyklus hinweg beurteilen zu können, werden umweltrelevante Produktdaten über die Wertschöpfungskette hinweg ausgetauscht werden. Verbraucher fragen immer öfter nach Informationen zur Qualität von Produkten, zu den sozialen Standards bei Herstellung und Lieferung sowie dem Umwelteinfluss von Produkt und

\section{»Ein zentrales Problem bei der Be- kämpfung des Klimawandels ist, dass Emissionen keinen allgemein anerkannten Preis haben. «}

Produktion. Puma veröffentlichte 2011 die erste ökologische Gewinn- und Verlustrechnung - und bezifferte den durch das Unternehmen verursachte Umweltschaden auf etwa 145 Millionen Euro [16]. Angesichts der gestiegenen Erwartung an unternehmerische Verantwortung und eines höheren Umwelt- und Problembewusstseins ist damit zu rechnen, dass in einigen Jahren die ökologische Gewinn- und Verlustrechnung ebenso selbstverständlich sein wird wie die finanzielle. Insbesondere die Nutzung fossiler Energien wird dann Gewicht haben. Das Beispiel Puma zeigt allerdings auch, dass die Hersteller allein die Daten gar nicht verfügbar machen können. Der Umwelteinfluss von Pumas eigenem Betrieb macht nur etwa ein Zehntel des gesamten ökologischen Fußabdrucks aus - $90 \%$ davon liegen in der Lieferkette. Das bedeutet, dass davon auszugehen ist, dass von den "global brands“ ausgehend der Druck zu mehr Transparenz der ökologischen und sozialen Standards entlang der gesamten Lieferkette entstehen wird. 


\section{Lessons Learned}

- Die Verknappung fossiler Rohstoffe erfolgt langsamer als das Erreichen anderer globaler ökologischer Grenzen, z. B. des Klimawandels.

- Der Umbau in eine postfossile Gesellschaft wird daher insbesondere politisch getrieben erfolgen.

- Durch massive Veränderungen in den Werthaltungen, eine zunehmende internationale Vernetzung und die schwer berechenbare Wirkung von Einzelereignissen (wie der Reaktorkatastrophe von Fukushima) kann dieser Umbau schneller als bisher vermutet erfolgen.

- Unternehmen sollten sich daher sehr früh mit den Merkmalen einer post-fossilen Wirtschaft auseinandersetzen und strategisch darauf reagieren.

- Zentrale Ansatzpunkte sind dabei eine erhöhte unternehmensinterne und wertschöpfungskettenweite Transparenz der $\mathrm{CO}_{2}$-Intensität, eine frühe Risikoanalyse des Geschäftsmodells, ein optimiertes Energiemanagement und eine vorausschauende Produktentwicklung.

\section{Was Unternehmen schon heute tun können}

Wenngleich fossile Energieträger noch Jahrzehnte lang vorhanden sein werden, können Unternehmen schon heute erste Schritte in eine post-fossile Zukunft tun - und dabei sogar Kosten senken und die Wettbewerbsfähigkeit erhöhen. Die Praxis zeigt nämlich, dass gerade die innovativsten und erfolgreichsten Unternehmen hier aktiv werden und Nachhaltigkeit als Wertschöpfungstreiber zu nutzen verstehen. [1]

\section{Transparenz erhöhen:}

\section{Energie-Verbräuche und $\mathrm{CO}_{2}$-Emissionen}

Obwohl es trivial klingt, in der Praxis mangelt es hier oft noch: Transparenz der betrieblichen Energie- und Emissionsdaten ist der erste wichtige Schritt, bevor weitere Maßnahmen adressiert werden können. Viele Unternehmen kennen ihre Energieverbrauchs- bzw. Emissionsdaten nur auf sehr aggregiertem Niveau und können weder die betrieblichen Energieströme noch die zeitlichen Verläufe abbilden. Beides zu kennen erlaubt aber eine gezielte Optimierung mit erheblichem Kostensenkungspotenzial, z. B. durch Kosten-NutzenAnalysen für Effizienzmaßnahmen bestimmter Verbrauchergruppen oder durch ein optimiertes Lastenmanagement.

\section{Energiemanagement optimieren}

Für die Optimierung des Energiemanagements gibt es oft einen klaren Business Case, da Verbrauchsreduktion direkt mit der Kostenreduktion korreliert. Obwohl die Unternehmen im Bereich Effizienzmaßnahmen schon viel getan haben, gibt es oft noch immer große Verbesserungspotenziale in Produktion, Verwaltung, Logistik oder in der IT. Unternehmen sparen hier sogar doppelt: bei der Energie-Beschaffung und bei den Zertifikaten für die $\mathrm{CO}_{2}$-Emissionen.

Aber auch durch die Energiebeschaffung kann die Abhängigkeit von Energiepreissteigerungen und fossilen Quellen gesenkt werden, zum Beispiel durch den Bezug von Strom aus erneuerbaren Energien. Immer mehr Unternehmen gehen dazu über, ihren Strom selbst zu produzieren. Laut einer DIHK-Umfrage erzeugen mittlerweile schon $13 \%$ der deutschen Unternehmen ihren eigenen Strom, noch einmal weitere $16 \%$ planen, dies künftig zu tun [7].

Risikoanalyse des Geschäftsmodells Entwicklung neuer Dienstleistungen

Der Übergang in eine post-fossile Gesellschaft wird mehrere Jahrzehnte dauern. Das gibt die Gelegenheit, langfristige strategische Planungen sukzessive anhand der Merkmale post-fossilen Wirtschaftens zu überprüfen bzw. daran auszurichten. Wie wichtig ist der Einfluss fossiler Energieträger, welche Rolle spielt deren Verfügbarkeit und Preis für den wirtschaftlichen Erfolg? Gibt es Möglichkeiten, das Geschäftsmodell zum Beispiel durch eine Erhöhung des Dienstleistungsangebots zu verändern? Wo können neue Wertschöpfungsmöglichkeiten erschlossen werden, die auf niedrigen Einsatz von Energie und Ressourcen bei hohem Nutzen für Verbraucher setzen, die sich in geschlossene Stoffkreisläufe einpassen und lokale Beschaffungsprozesse unterstützen?

Verbesserung des ökologischen Fußabdrucks von Produkten

Die ökologischen Nebenwirkungen von Produkten und Dienstleistungen sind komplex und wirklich genau nur in einer System-Betrachtung möglich. Um dennoch schon einen ersten Anhaltspunkt für die ökologische Qualität eines Produkts zu erhalten, wird vermehrt der „Carbon Footprint" von Produkten errechnet, der die entlang des Produkt-Lebenszyklus freigesetzte Menge an Treibhausgasen berechnet. 
Einer Umfrage des Carbon Disclosure Project von 2012 zufolge nimmt die Zahl der großen Unternehmen, die Klimaschutzziele in die Geschäftsstrategie integrieren, weiter zu. Von den befragten Global-500-Unternehmen (von denen $81 \%$ die Umfrage beantworteten), gaben $78 \%$ an, Ziele zur Emissionsreduktion in die Strategie integriert zu haben (gegenüber 68 \% im Jahr 2011) [3]. Da sehr viele Unternehmen in der einen oder anderen Form Teil der Lieferkette großer Marken sind, wird es auch für sie immer wichtiger, die Anforderungen der Markenhersteller und Händler zu kennen und ihnen zu genügen.

Fazit: Es gibt hinreichend gute Argumente für Unternehmen, die Abhängigkeit von fossilen Energieträgern auch heute schon kritisch zu beleuchten und die Randbedingungen post-fossilen Wirtschaftens in der Strategie zu verankern.

\section{» Unternehmen können schon heute erste Schritte in eine post-fossile Zukunft tun. "}

\section{Links und Literatur}

1. Berg, C./Hack, S. (2012): Sustainability Driving Innovation and Value Creation, in: Busines+Innovation, Vol. 3. Jg. (2012), Nr. 3, S. 26-32.

2. Deutsche Rohstoffagentur (DERA) in der Bundesanstalt für Geowissenschaften und Rohstoffe (2011): Reserven, Ressourcen und Verfügbarkeit von Energierohstoffen 2011, Hannover.

3. Carbon Disclosure Project (2012), online: https://www. cdproject.net/en-US/Results/Documents/2012-G500-executivesummary.pdf, Stand: o. A., Abruf: 30.11.2012

4. Carbontracker (2012): Unburnable Carbon - Are the World's Financial Markets Carrying a Carbon Bubble? Online: http://

\section{Autoren}

\section{Prof. Dr. Christian Berg}

ist Chief Sustainability Architect innerhalb des Global Services Sustainability Hub der SAP. Zudem ist er Honorarprofessor für „Nachhaltigkeit und Globalen Wandel" an der TU Clausthal sowie wiederkehrender Gastprofessor für "Corporate Sustainability" an der Universität des Saarlandes.

E-Mail:ch.berg@sap.com

\section{Prof. Dr. Uwe Schneidewind}

ist Präsident und wissenschaftlicher Geschäftsführer des Wuppertal Institut für Klima, Umwelt, Energie. Er ist zudem Inhaber der Professur für Innovationsmanagement und Nachhaltigkeit an der Bergischen Universität Wuppertal.

E-Mail: uwe.schneidewind@wupperinst.org www.carbontracker.org/wp-content/uploads/downloads/2012/08/Unburnable-Carbon-Full1.pdf, Stand: o. A., Abruf: 26.11 .2012 .

5. Citigroup (2012), online: https://www.citigroupgeo.com/pdf/ SEUNHGJJ.pdf, Stand: 12.02.2012, Abruf: 26.11.2012.

6. Deutsche Schutzvereinigung für Wertpapierbesitz: Rede von Ulrich Hocker, online: http://www.dsw-info.de/Analyse-derPrognoseberichte-d.1890.0.html\#c3769, Stand: o. A., Abruf: 26.11.2012.

7. DIHK - Deutscher Industrie- und Handelskammertag (2012): IHK-Energiewende-Barometer 2012, online: http://www.dihk. de/presse/meldungen/2012-12-17-energiewende-barometer, Stand: 17.12.2012, Abruf: 21.12.2012.

8. Exxon Mobil: 2010 The Outlook for Energy - a View to 2030, online: http://www.exxonmobil.com/Corporate/files/news_ pub_eo_2010.pdf, Stand: o. A., Abruf: 30.11.2012.

9. ifeu - Institut für Energie- und Umweltforschung (2012): Volkswirtschaftliche Effekte der Energiewende, Heidelberg.

10. Internet World Stats - Usage and Population Statistics (2012), online: http://www.internetworldstats.com/stats.htm, Stand: o. A., Abruf: 30.11.2012.

11. Internationale Energieagentur (2010): World Energy Outlook 2010. Executive Summary, Paris.

12. Internationale Energieagentur (2012), online: Video on press conference, http://www.worldenergyoutlook.org/multimedia/ videocontent/name,33415,en.html, Stand: o. A., Abruf: 30.11.2012.

13. IWR - Internationales Wirtschaftsforum Regenerative Energien (2012), online http://www.iwr.de, Stand: o. A., Abruf: 30.11.2012.

14. Patagonia, online: www.patagonia.com, Stand: o. A., Abruf: 30.11.2012.

15. Presse- und Informationsamt der Bundesregierung (2012): Dialog über Deutschlands Zukunft. Ergebnisbericht des Expertendialogs der Bundeskanzlerin, Berlin (online: https://www.dialogueber-deutschland.de/SharedDocs/Downloads/DE/Ergebnisbericht/Kurzfassung-gesamt.pdf?_blob=publicationFile\&v=1)

16. Puma (2011): Puma's Environmental Profit and Loss Account for the year ended 31 December 2010, online: http://about. puma.com/wp-content/themes/aboutPUMA_theme/financialreport/pdf/EPL080212final.pdf, Stand: ???, Abruf: 21.12.2012.

17. Rifkin, J. (2007): Access - Das Verschwinden des Eigentums. Warum wir weniger besitzen und mehr ausgeben, Frankfurt.

18. Servatius, H.-G., Schneidewind, U., Rohlfing, D. (2012): Smart Energy - Wandel zu einem nachhaltigen Energiesystem, Heidelberg.

19. Shell (2008): Energy Scenarios 2050, online: http://www.shell. com/home/content/future_energy/scenarios/2050/, Stand: o. A., Abruf: 30.11.2012.

20. Wissenschaftlicher Beirat der Bundesregierung Globale Umweltveränderungen (WBGU) (2009): Kassensturz für den Weltklimavertrag - Der Budgetansatz, Sondergutachten 2009, Berlin.

21. Wissenschaftlicher Beirat der Bundesregierung Globale Umweltveränderungen (WBGU) (2011): Welt im Wandel - Gesellschaftsvertrag für eine Große Transformation, Hauptgutachten 2011, Berlin.

22. Wilke, J. (2002): Entwicklungsstufen und Determinanten der Kommunikationsgeschichte, in: Handel, K. (Hrsg.), Kommunikation in Geschichte und Gegenwart, Freiburg.

23. World Bank (2012): $4^{\circ}$ Turn Down the Heat. Why a $4^{\circ} \mathrm{C}$ World Must be Avoided, Washington D.C.

24. Yerdle (2012), online: www.yerdle.com, Stand: o. A., Abruf: 25.11.2012. 